\title{
ON THE EXISTENCE OF EXACTLY $(2,1)$ MAPS
}

\author{
R. E. SMITHSON
}

\begin{abstract}
The following two theorems concerning the existence of exactly 2 to 1 maps are proved. If $Y$ is a continuum such that each nondegenerate subcontinuum contains a cutpoint, then there does not exist a continuum $X$ and an exactly 2 to 1 map on $X$ onto $Y$. Further, if $X$ is an arcwise connected continuum and $Y$ is a nested continuum, then there does not exist an exactly 2 to 1 map on $X$ onto $Y$.
\end{abstract}

1. Introduction. In 1940 O. G. Harrold [5] showed that the arc was not the image of an exactly $n$-to- 1 map defined on a continuum. He also showed that an exactly $n$-to- 1 image of a finite graph contains a circle. The natural question to ask here is which continua are the images of some continuum under an exactly $n$-to- 1 map. So far the answers to this question are generally given in the negative as in the above-mentioned result of Harrold [5]. Recently Nadler and Ward [6] proved that if $Y$ is a continuum such that every subcontinuum of $Y$ contains an endpoint, then $Y$ is not the image of a continuum under an exactly $n$-to- 1 map. They were also able to show that if $Y$ is a nonhereditarily unicoherent continuum, then there is a continuum $X$ and an exactly $n$-to- 1 map on $X$ onto $Y$.

In the following, a continuum is a compact, connected $T_{2}$-space, and a map is a continuous function. Then a map $f$ is exactly $(n, 1)$ in case the inverse of each point in the range contains exactly $n$ points. A cutpoint of a continuum $Y$ is an element $p$ such that $Y-\{p\}$ is not connected. Then an arc is a continuum which contains exactly two noncutpoints. In the sequel we shall be primarily interested in exactly $(2,1)$ maps.

2. Spaces with cutpoints. In this section we shall investigate the situation when the continuum $Y$ is rich in cutpoints. First we present the following example.

EXAMPle 1. Let $X$ be the circle $C_{1}$, the twig at $x_{1}$ from $C_{1}$ to $x_{2}$ and $C_{2}, C_{3}$ two circles which are tangent at $x_{2}$ and do not meet $C_{1}$, and let $Y$ be the two circles $Y_{1}, Y_{2}$ which are tangent at $y_{1}$ (see Figure 1).

Define $f$ by: First, $f\left(x_{1}\right)=f\left(x_{2}\right)=y_{1}$. Then choose $f$ so that it is 1-to-1, and continuous on $C_{1}$ onto $Y_{1}-\left\{y_{1}\right\}$ and so that $f$ is 1-to-1, and continuous on $L_{1}-\left\{x_{1}, x_{2}\right\}$ onto $Y_{1}$. Finally, $f$ maps $C_{1}-x_{2}$ and $C_{3}-x_{2}$ in a 1-to- 1 continuous manner onto $Y_{2}-y_{1}$. Then $f$ is a continuous, exactly $(2,1)$ map on $X$ onto $Y$.

Next we give a lemma which is crucial to the main result of this section.

LEMMA 2.1. Let $f: X \rightarrow Y$ be an exactly $(2,1)$ map on the continuum $X$ onto the continuum $Y$. If $Y$ contains a cutpoint, then there are proper subcontinua $X_{0}, Y_{0}$ respectively such that $f$ restricted to $X_{0}$ is exactly $(2,1)$ onto $Y_{0}$.

Received by the editors March 20, 1984 and, in revised form, April 2, 1985.

1980 Mathematics Subject Classification. Primary 54C10, 54F20.

Key words and phrases. Exactly $n$-to-1 maps, continua, cutpoints, nested continua. 

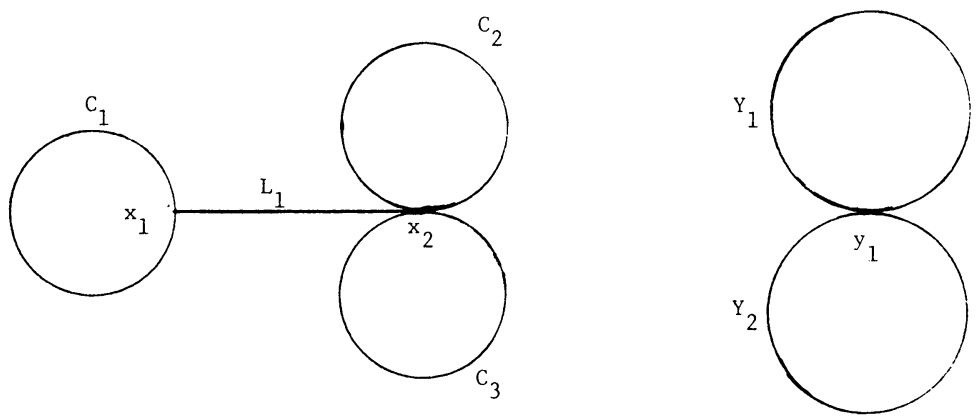

\section{FIGURE 1}

Proof. Let $p$ be a cutpoint of $Y$ and let $Y-\{p\}=A \cup B$, where $A, B$ are nonempty separated sets. Then $f^{-1}(p)=\left\{x_{1}, x_{2}\right\}$ and let $M$ be a subcontinuum of $X$ which is irreducible about $x_{1}, x_{2}$. Then $M-\left\{x_{1}, x_{2}\right\}$ is connected. Thus there is a component $C_{0}$ of $X-\left\{x_{1}, x_{2}\right\}$ which contains $M-\left\{x_{1}, x_{2}\right\}$. Now $f\left(C_{0}\right)$ is contained in either $A$ or $B$. So assume that $f\left(C_{0}\right) \subset A$. Also note that $\bar{C}_{0}=C_{0} \cup\left\{x_{1}, x_{2}\right\}$. Next suppose that $x$ is an element of $f^{-1}(A)$ and let $C_{x}$ be the component of $X-\left\{x_{1}, x_{2}\right\}$ which contains $x$. Then $\bar{C}_{x} \cap\left\{x_{1}, x_{2}\right\} \neq \varnothing$ (see [3, Theorem 4.A.12, p. 105]) and $f\left(C_{x}\right) \subset A$.

Let $\mathbf{C}_{1}=\left\{\bar{C}_{x} \mid x \in f^{-1}(A)\right.$ and $\left.x_{1} \in \bar{C}_{x}\right\}$ and let $\mathbf{C}_{2}=\left\{\bar{C}_{x} \mid x \in f^{-1}(A)\right.$ and $\left.x_{2} \in \bar{C}_{x}\right\}$. Then $X_{0}=\bigcup\left(\mathbf{C}_{1} \cup \mathbf{C}_{2}\right)=f^{-1}(p \cup A)$ is closed and connected. Thus $X_{0}$ is a subcontinuum of $X$ which contains $C_{0}$ and $f$ restricted to $X_{0}$ maps onto $Y_{0}=A \cup p$. Further, if $y \in A$ and $f^{-1}(y)=\left\{x, x^{\prime}\right\}$, then $\left\{x, x^{\prime}\right\} \in X_{0}$ and thus $\left.f\right|_{X_{0}}$ is exactly $(2,1)$ on $X_{0}$. Moreover, $Y_{0}$ is a proper subcontinuum of $Y$ since $B \neq \varnothing$, and we are done.

From Lemma 2.1 we get

THEOREM 2.2. If $Y$ is a continuum such that each nondegenerate subcontinuum of $Y$ contains a cutpoint, then there does not exist a continuum $X$ and an exactly $(2,1)$ map on $X$ onto $Y$.

ProOF. Suppose there is a continuum $X$ and an exactly $(2,1)$ map on $X$ onto $Y$. Let $A$ be a maximal nest of subcontinua of $X$ such that $\left.f\right|_{A}$ is exactly $(2,1)$ for all $A \in A$. Then $X_{0}=\bigcap A$ is a subcontinuum of $X$ and $\left.f\right|_{X_{0}}$ is exactly $(2,1)$. Now $Y_{0}=f\left(X_{0}\right)$ is a nondegenerate subcontinuum of $Y$ and, hence, contains a cutpoint. Then an application of Lemma 2.1 gives a contradiction to the maximality of $A$.

The assumption that $f$ was exactly $(2,1)$ was used strongly in the proof of Lemma 2.1 and the following example shows that the techniques used in that proof will not work for exactly $(n, 1)$ maps when $n>2$.

EXAMPLE 2. Let $X$ be two thetas joined at a point as shown in Figure 2, and let $Y$ be a "figure 8 ".

To define $f$, let $f\left(x_{1}\right)=f\left(x_{2}\right)=f\left(x_{3}\right)=p$ and let each of the open arcs with endpoints $x_{1}, x_{2}$ map homeomorphically onto $C_{1}-\{p\}$. Also, let the open arcs with endpoints $x_{2}, x_{3}$ map homeomorphically onto $\mathbf{C}_{2}-\{p\}$. Then $f$ is an exactly $(3,1)$ map onto $Y$. However, if $C$ is a component of $X-f^{-1}(p)$, then $\bar{C} \operatorname{does}$ not contain 

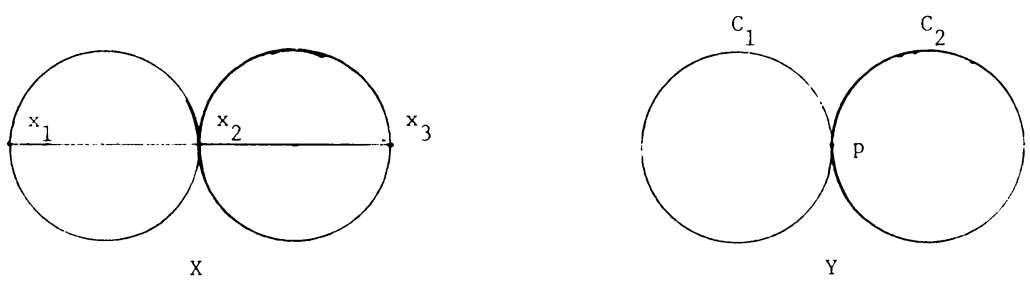

FIGURE 2

all of $f^{-1}(p)$. Thus the technique used in the proof of Lemma 2.1 will not yield a proper subcontinuum of $X$ on which $f$ is exactly $(3,1)$. There are subcontinua of $X$ on which $f$ is $(2,1)$ but so far a general method of finding such subcontinua has not been found. We are left with the following question.

Question 1. Can Theorem 2.2 be extended to include exactly $(n, 1)$ maps?

An immediate corollary to Theorem 2.2 is Nadler and Ward's [6] theorem for $(2,1)$ maps. In Corollary 2.3 a point $e \in Y$ is an endpoint if $e$ has arbitrarily small neighborhoods with one point boundary.

COROLLARY 2.3. If $Y$ is a continuum such that every subcontinuum of $Y$ contains an endpoint, then there does not exist a continuum $X$ and an exactly $(2,1)$ map on $X$ onto $Y$.

3. Arcwise connected shapes. If we put further restrictions on the domain space $X$, we can obtain other results. For example, it is known that there is no exactly $(2,1)$ map defined on a closed $n$-cell for $n=1,2$ or 3 . In this section we consider the case when $X$ is arcwise connected.

In the following, a space is nested in case it is an arcwise connected, Hausdorff space in which the union of a nest of arcs is contained in an arc.

THEOREM 3.1. Let $X$ be an arcwise connected continuum and $Y$ a nested continuum. Then there does not exist an exactly $(2,1)$ map on $X$ onto $Y$.

Proof. Suppose that $f: X \rightarrow Y$ is exactly $(2,1)$ and let $p \in Y$. Let $f^{-1}(p)=$ $\left\{x_{1}, x_{2}\right\}$ and let $A_{1}$ be an arc in $X$ with endpoints $x_{1}, x_{2}$. We know from Harrold's result [4] that $f$ restricted to $A_{1}$ is not exactly $(2,1)$. Thus, let $x_{3} \in A_{1}$ be such that $f^{-1}\left(f\left(x_{3}\right)\right) \cap A_{1}=\left\{x_{3}\right\}$, and let $\left[p, f\left(x_{3}\right)\right]$ denote the unique arc in $Y$ with endpoints $p, f\left(x_{3}\right)$. Then, by a result of Harris [3], $f\left(A_{1}\right)$ is arcwise connected and hence, $\left[p, f\left(x_{3}\right)\right] \subset f\left(A_{1}\right)$. Next we shall show that $f\left(A_{1}\right) \subset\left[p, f\left(x_{3}\right)\right]$. Suppose not; that is let $z \in A_{1}$ be such that $f(z) \notin\left[p, f\left(x_{3}\right)\right]$. Then there is an open interval $\left(z_{1}, z_{2}\right)$ in $A_{1}$ such that $f\left(\left(z_{1}, z_{2}\right)\right) \cap\left[p, f\left(x_{3}\right)\right]=\varnothing$ but $f\left(z_{1}\right)$ and $f\left(z_{2}\right)$ are in $\left[p, f\left(x_{3}\right)\right]$. We may assume that $\left[z_{1}, z_{2}\right] \subset\left[x_{1}, x_{3}\right]$ in $A_{1}$. Now suppose that $f\left(z_{1}\right) \neq f\left(z_{2}\right)$; then since $f\left(z_{1}\right), f\left(z_{2}\right) \in\left[p, f\left(x_{3}\right)\right]$, the unique arc $\left[f\left(z_{1}\right), f\left(z_{2}\right)\right] \subset\left[p, f\left(x_{3}\right)\right]$ and so $f\left(z_{1}, z_{2}\right)$ meets $\left[p, f\left(x_{3}\right)\right]$ which contradicts the choice of $z_{1}, z_{2}$. Thus $f\left(z_{1}\right)=f\left(z_{2}\right)$. The image of the arc $\left[x_{2}, x_{3}\right]$ must contain $\left[p, f\left(x_{3}\right)\right]$ and thus there is also a point $x^{\prime}$ in the arc $\left[x_{2}, x_{3}\right]$ in $A_{1}$ such that $f\left(x^{\prime}\right)=f\left(z_{1}\right)=f\left(z_{2}\right)$. This contradicts the fact that $f$ is exactly $(2,1)$ on $X$. We have shown that each of the intervals $\left[x_{1}, x_{3}\right]$ and $\left[x_{2}, x_{3}\right]$ in $A_{1}$ maps onto $\left[p, f\left(x_{3}\right)\right]$ and $f$ is exactly $(2,1)$ on $A-\left\{x_{3}\right\}$. Moreover, the above argument applies to any arc $A \subset X$ whose endpoints map onto the same point. 
Now let $\mathcal{A}$ be a maximal nest of arcwise connected, closed subsets of $X$ which satisfy the following conditions: (i) for each $A \in A$, there is an $x_{a} \in A$ such that $f$ is exactly $(2,1)$ on $A-\left\{x_{a}\right\}$; (ii) $f^{-1}\left(f\left(x_{a}\right)\right) \cap A=\left\{x_{a}\right\}$; and (iii) there is a $p \in Y$ such that $f(A)=\left[p, f\left(x_{a}\right)\right]$. By the above results we see that there are nonempty collections which satisfy the three conditions. Let $A_{0}=\bigcup A$ and $X_{0}=\bar{A}_{0}$. The collection $\{f(A) \mid A \in \mathcal{A}\}$ is a nest of arcs in $Y$ and so there is a $y_{m} \in Y$ such that $f\left(A_{0}\right) \subset\left[p, y_{m}\right]$ and $\overline{f\left(A_{0}\right)}=\left[p, y_{m}\right]$. Further, $X_{0} \subset f^{-1}\left(\left[p, y_{m}\right]\right)$.

Now let $f^{-1}\left(y_{m}\right)=\{s, z\}$ and let $x_{1} \in f^{-1}(p) \cap A_{0}$. If $A$ is an arc with endpoints $x_{1}, s$, then $f(A)$ contains $\left[p, y_{m}\right]$. Then, since $f^{-1}\left(\left[p, y_{m}\right)\right) \subset A_{0} \subset X_{0}$, there is a nest $z_{\gamma}, \gamma \in \Gamma$, in $A_{0} \cap A$ such that $f\left(z_{\gamma}\right) \rightarrow y_{m}$. We may assume that $z_{\gamma} \rightarrow z$ in $A \cap X_{0}$, and, if $z \notin\{s, t\}$, then $f$ is not exactly $(2,1)$. Hence, either $s$ or $t$ is in $A \cap X_{0}$. We assume that $s \in X_{0}$ and that $t \notin X_{0}$. We let $A_{1}$ be an arc with endpoints $s, t$. Then there is a point $a_{1} \in A_{1}$ such that $f$ is exactly $(2,1)$ on $A-\left\{a_{1}\right\}$ and $f^{-1}\left(f\left(a_{1}\right)\right) \cap A_{1}=\left\{a_{1}\right\}$. Now $f$ is exactly $(2,1)$ on $A_{0}$ and so $f$ is exactly $(2,1)$ on $X_{0} \cup A_{1}-\left\{a_{1}\right\}$. If $f$ is exactly $(2,1)$ on $X_{0} \cup A_{1}$, then $f$ is an exactly $(2,1)$ map on a continuum onto an arc, which is a contradiction. But $X_{0} \cup A_{1}$ properly contains each member of $A$ contrary to the maximality of $A$. Finally, if both $x, t \in X_{0}$ we again have an exactly $(2,1)$ map on a continuum $X_{0}$ onto an arc. Thus there can be no such map on $X$.

We conclude with two questions.

Question 2. Can the hypothesis that $X$ and $Y$ be continua in Theorem 3.1 be weakened? In particular, suppose that $X$ is an arcwise connected $T_{2}$-space and $Y$ a nested space. Then is there an exactly $(2,1)$ map on $X$ onto $Y$ ?

Question 3. Is there an exactly $(n, 1)$ version of Theorem 3.1 ?

\section{REFERENCES}

1. C. O. Christenson and W. L. Voxman, Aspects of topology, Marcel Dekker, New York, 1977.

2. P. Civin, Two-to-one mappings of manifolds, Duke Math. J. 10 (1943), 49-57.

3. J. K. Harris, Order structures for certain acyclic topological spaces, Thesis, Univ. of Oregon, 1962.

4. O. G. Harrold, The non-existence of a certain type of continuous transformation, Duke Math. J. 5 (1939), 789-793.

5. _ Exactly $(k, 1)$ transformations on connected linear graphs, Amer. J. Math. 62 (1940), 823-834.

6. S. B. Nadler, Jr. and L. E. Ward, Jr., Concerning exactly $(n, 1)$ images of continua, Proc. Amer. Math. Soc. 87 (1983), 351-354.

7. J. H. Roberts, Two-to-one transformations, Duke Math. J. 6 (1940), 256-262. 82071

Department of MAThematics, University of Wyoming, LARAmie, Wyoming 